\title{
Eigenvalue Based Approach for Global Consensus in Multiagent Systems with Nonlinear Dynamics
}

\author{
Wei Qian' ${ }^{1}$ and Lei Wang ${ }^{2}$ \\ ${ }^{1}$ School of Electrical Engineering and Automation, Henan Polytechnic University, Jiaozuo, Henan 454000, China \\ ${ }^{2}$ School of Mathematics and Systems Science and LMIB, Beihang University, Beijing 100191, China \\ Correspondence should be addressed to Wei Qian; qwei@hpu.edu.cn
}

Received 25 March 2014; Accepted 4 May 2014; Published 14 May 2014

Academic Editor: Zidong Wang

Copyright ( 2014 W. Qian and L. Wang. This is an open access article distributed under the Creative Commons Attribution License, which permits unrestricted use, distribution, and reproduction in any medium, provided the original work is properly cited.

This paper addresses the global consensus of nonlinear multiagent systems with asymmetrically coupled identical agents. By employing a Lyapunov function and graph theory, a sufficient condition is presented for the global exponential consensus of the multiagent system. The analytical result shows that, for a weakly connected communication graph, the algebraic connectivity of a redefined symmetric matrix associated with the directed graph is used to evaluate the global consensus of the multiagent system with nonlinear dynamics under the common linear consensus protocol. The presented condition is quite simple and easily verified, which can be effectively used to design consensus protocols of various weighted and directed communications. A numerical simulation is also given to show the effectiveness of the analytical result.

\section{Introduction}

Cooperative collective behavior in networked systems of autonomous agents has received a great deal of attention in the past decade. This is partially due to the growing interest in understanding group behaviors in nature, such as flocking and swarming, and also due to its broad applications of multiagent systems in many areas in sensor networks [1] and mobile robots [2], to name a few. In all cases, the aim is to control a group of agents connected through a communication network. Consensus problem that enables all agents to reach an agreement on a certain value of interest is a fundamental issue of controlling multiagent systems.

With the fundamental "nearest neighbor rule," various models have been introduced to study the consensus problem. In [3], Vicsek et al. proposed a simple model for the phase transition of a group of autonomous agents and demonstrated by simulations the headings of agents converge to a common value. Jadbabaie et al. [4] provided a theoretical explanation for the collective behavior observed in [3] by using graph theory. In particular, Olfati-Saber and Murray presented a general framework of the consensus problem for multiagent systems with fixed or switching topologies and established the relationship between the algebraic connectivity and the convergence for a balanced directed network [5]. Moreover, some more relaxable conditions were presented for consensus with switching topology $[6,7]$. Thereafter, many researchers extended these earlier works and proposed different protocols for agents that process second-order and higher-order dynamics in linear multiagent systems [818]. Recently, the consensus problem in multiagent systems with nonlinear dynamics has been investigated [19-25]. In particular, a passivity-based design tool was introduced to reach the velocity consensus among agents in [19]. A finitetime consensus algorithm was proposed to achieve consensus by using nonsmooth gradient flows [21]. By constructing a Lyapunov function, sufficient conditions in the form of generalized algebraic connectivity were established for reaching local and global consensus in [26].

In this paper, we present a sufficient condition of global consensus for multiagent systems with nonlinear dynamics by using a Lyapunov function and some graph theory techniques. A simple linear protocol is designed to generalize the tools developed for undirected graph in order to make 
them applicable to directed graphs. Similar to the idea in [26], the algebraic connectivity of a redefined symmetric matrix associated with the directed graph is used to evaluate the consensus of the considered system. The obtained result is quite simple and powerful, especially for those multiagent systems containing spanning trees.

The rest of this paper is organized as follows. Section 2 presents some preliminaries. In Section 3, we derive a sufficient condition to guarantee the global consensus under the designed protocol. A numerical validation is given to show the effectiveness of the presented result in Section 4. Section 5 concludes the investigation.

\section{Preliminaries}

Suppose that the multiagent system under consideration consists of $N$ identical agents, each of which evolves as a nonlinear behavior and is described by the equation

$$
\dot{x}_{i}(t)=f\left(x_{i}(t)\right)+B u_{i}(t), \quad 1 \leq i \leq N,
$$

where $x_{i}(t) \in R^{n}$ and $u_{i} \in R^{p}$ denote the state and the control input of agent $i$, respectively, $f: R^{n} \rightarrow R^{n}$ is continuously differentiable, describing the intrinsic nonlinear dynamics of the isolated agent, and constant matrix $B \in R^{n \times p}$ is the input matrix. The consensus protocol in multiagent system (1) considered in $[27,28]$ is chosen as

$$
u_{i}(t)=-c K \sum_{j=1}^{N} l_{i j} x_{j}(t),
$$

where $c$ is the overall coupling strength, $K \in R^{p \times n}$ is the feedback gain matrix to be determined, and the communication topology among agents is represented by digraph $\mathscr{G}$ and described in a matrix form by the Laplacian $L=$ $\left(l_{i j}\right) \in R^{N \times N}$. The Laplacian matrix of digraph $\mathscr{G}$ is defined as follows: if there is a directed connection from agent $i$ to agent $j(j \neq i)$, then $l_{i j}<0$; otherwise, $l_{i j}=0$, and the diagonal entries of matrix $L$ are defined by $l_{i i}=-\sum_{j=1, i \neq j}^{N} l_{i j}$. It is noted that network (1) is said to be a multiagent system with asymmetrically coupled identical agents if the Laplacian matrix $L$ is not assumed to be symmetric and irreducible.

Clearly, under the diffusive condition, if a consensus is achieved, the solution $s(t)$ of system (1) is expected to be a possible trajectory of an individual agent satisfying $\dot{s}(t)=f(s(t))$, where $s(t)$ can be an equilibrium or a periodic or chaotic orbit.

Let

$$
\begin{aligned}
& X(t)=\left(x_{1}^{T}(t), x_{2}^{T}(t), \ldots, x_{N}^{T}(t)\right)^{T} \in R^{n N}, \\
& F(X)=\left(f_{1}^{T}\left(x_{1}\right), \ldots, f_{N}^{T}\left(x_{N}\right)\right)^{T} \in R^{n N}
\end{aligned}
$$

then the multiagent system (1) with protocol (2) can be written in a block form as

$$
\dot{X}(t)=F(X)-c(L \otimes B K) X(t),
$$

where the notation $\otimes$ represents the Kronecker product. For the subsequent analysis, we decompose $L$ into $L=L^{+}+L^{-}$, where symmetric matrix $L^{+}=\left(l_{i j}^{+}\right)_{N \times N}$ and antisymmetric matrix $L^{-}=\left(l_{i j}^{-}\right)_{N \times N}$ satisfy the zero-row-sum condition with nondiagonal entries

$$
\begin{aligned}
& l_{i j}^{+}=l_{j i}^{+}=\frac{1}{2}\left(l_{i j}+l_{j i}\right) \\
& l_{i j}^{-}=-l_{j i}^{-}=\frac{1}{2}\left(l_{i j}-l_{j i}\right) .
\end{aligned}
$$

Throughout this paper, two important hypotheses are introduced.

Hypothesis 1 (H1). Suppose that the directed graph $\mathscr{G}$ is weakly connected. That is, there exists a path between any two agents in graph $\mathscr{G}$ if one replaces all the directed edges of graph $\mathscr{G}$ with undirected edges.

Noting that all nondiagonal entries of matrix $L^{+}$are nonnegative, then $L^{+}$can be regarded as the Laplacian matrix associated with an undirected graph $\widetilde{\mathscr{G}}$. Moreover, under (H1), $L^{+}$has an eigenvalue 0 with multiplicity 1 and the eigenvector $\xi=(1 / \sqrt{N})(1,1, \ldots, 1)^{T} \in R^{N}$ and the algebraic connectivity (the second smallest eigenvalue), denoted by $\lambda_{2}\left(L^{+}\right)$, is positive [29].

Hypothesis $2(\mathrm{H} 2)$. Suppose that there exist a symmetric matrix $P \in R^{n \times n}>0$ and constants $\alpha>0$ and $\beta>0$ such that, for all $i, j=1, \ldots, N$,

$$
\begin{aligned}
& \left(f\left(x_{i}\right)-f\left(x_{j}\right)\right)^{T} P X_{i j}(t)-\alpha X_{i j}^{T}(t) P B B^{T} P X_{i j}(t) \\
& \quad+\beta X_{i j}^{T}(t) X_{i j}(t) \leq 0,
\end{aligned}
$$

where $X_{i j}(t)=x_{i}(t)-x_{j}(t)$.

As shown in [30], (H2) is solvable for many coupled limit-cycle or chaotic systems, such as Hindmarsh-Rose neuron models, Lorenz systems, and coupled double-scrolls. Considering a special case that $f\left(x_{i}\right)=A x_{i}$, then (6) reduces to the following linear matrix inequality:

$$
A^{T} P+P A-2 \alpha P B B^{T} P+2 \beta I_{n} \leq 0,
$$

or equivalently written as

$$
A^{T} P+P A-2 \alpha P B B^{T} P<0 .
$$

Recalling the result in [27], a necessary and sufficient condition for the existence of a $P>0$ to (8) is that $(A, B)$ is stabilizable. For nonlinear vector-valued function $f$ satisfying the Lipschitz condition, a feasible operation is to convert inequality (6) into some linear matrix inequalities; see (35)-(36) for details.

\section{Main Results}

For a multiagent system of coupled identical agents, a typical approach that handles the consensus issue is to investigate 
the stability of errors between the dynamics of an individual agent and the average dynamics [25]; that is,

$$
y_{i}(t)=x_{i}(t)-\frac{1}{N} \sum_{j=1}^{N} x_{j}(t)
$$

or equivalently written in a block form as

$$
Y(t)=\left(\Pi \otimes I_{n}\right) X(t)
$$

where $Y(t)=\left(y_{1}^{T}(t), \ldots, y_{N}^{T}(t)\right)^{T} \in R^{n N}$ is the error vector, $\Pi=I_{N}-\xi \xi^{T}$, and $I_{N}$ is an $N \times N$ identity matrix. Then we have the following results.

Theorem 1. Suppose that (H1) and (H2) hold. If

$$
\lambda_{2}(\widetilde{L}) \geq \frac{\alpha}{c}
$$

then the multiagent system (1) achieves global exponential consensus under the designed controller (2) and $K=B^{T} P$, where $\lambda_{2}(\widetilde{L})$ is the algebraic connectivity of matrix $\widetilde{L}=$ $\left(\widetilde{l}_{i j}\right)_{N \times N}$, which is a zero-row-sum symmetric matrix with nondiagonal entries

$$
\tilde{l}_{i j}=l_{i j}^{+}-\frac{1}{N}\left(l_{i i}^{-}+l_{j j}^{-}\right), \quad \forall i \neq j .
$$

Proof. Solving $(\mathrm{H} 2)$ to obtain a symmetric matrix $P>0$, then one can choose the Lyapunov function as

$$
V(t)=\frac{1}{2} X^{T}(t)(\Pi \otimes P) X(t) .
$$

Differentiating $V(t)$ along the trajectory of system (4) gives

$$
\begin{aligned}
\dot{V}(t)= & X^{T}(t)(\Pi \otimes P) F(X) \\
& -c X^{T}(t)(\Pi \otimes P)(L \otimes B K) X(t) \\
\triangleq & S_{1}(t)-c S_{2}(t),
\end{aligned}
$$

where the term $S_{1}(t)$ satisfies the following equality:

$$
S_{1}(t)=\frac{1}{N} \sum_{i=1}^{N} \sum_{j=1}^{N} x_{i}^{T}(t) P\left[f\left(x_{i}\right)-f\left(x_{j}\right)\right] .
$$

Renaming the sum $S_{1}(t) j$ by $i$ and vice versa yields

$$
\begin{aligned}
S_{1}(t) & =-\frac{1}{N} \sum_{i=1}^{N} \sum_{j=1}^{N} x_{j}^{T}(t) P\left[f\left(x_{i}\right)-f\left(x_{j}\right)\right] \\
& =\frac{1}{2 N} \sum_{i=1}^{N} \sum_{j=1}^{N} X_{i j}^{T}(t) P\left(f\left(x_{i}\right)-f\left(x_{j}\right)\right) .
\end{aligned}
$$

Substituting (6) into (16) obtains

$$
\begin{aligned}
S_{1}(t) \leq & \frac{1}{2 N} \sum_{i=1}^{N} \sum_{j=1}^{N} \alpha X_{i j}^{T}(t) P B B^{T} P X_{i j}(t) \\
& -\frac{1}{2 N} \sum_{i=1}^{N} \sum_{j=1}^{N} \beta X_{i j}^{T}(t) X_{i j}(t) \\
= & \alpha X^{T}(t)\left[\Pi \otimes\left(P B B^{T} P\right)\right] X(t) \\
& -\beta X^{T}(t)\left(\Pi \otimes I_{n}\right) X(t) .
\end{aligned}
$$

And the term $S_{2}(t)$ satisfies the equation

$$
\begin{aligned}
S_{2}(t) & =X^{T}(t)[(\Pi L) \otimes(P B K)] X(t) \\
& =\frac{1}{N} \sum_{i=1}^{N} \sum_{j=1}^{N} \sum_{k=1}^{N}\left(l_{i k}-l_{j k}\right) x_{i}^{T}(t) P B K x_{k}(t) .
\end{aligned}
$$

By the diffusive condition of the Laplacian matrix $L$, we have

$$
\sum_{i=1}^{N} \sum_{j=1}^{N} \sum_{k=1}^{N} l_{i k} x_{i}^{T}(t) P B K x_{i}(t)=0
$$

Also by renaming $j$ by $i$ and vice versa, one obtains

$$
\begin{aligned}
& \sum_{i=1}^{N} \sum_{j=1}^{N} \sum_{k=1}^{N} l_{i k} x_{j}^{T}(t) P B K x_{k}(t) \\
& =\sum_{i=1}^{N} \sum_{j=1}^{N} \sum_{k=1}^{N} l_{j k} x_{i}^{T}(t) P B K x_{k}(t) .
\end{aligned}
$$

Substituting (19)-(20) into $S_{2}(t)$ we obtain

$$
\begin{aligned}
S_{2}(t) & =\frac{1}{N} \sum_{i=1}^{N} \sum_{j=1}^{N} \sum_{k=1}^{N}\left(l_{i k}^{+}+l_{i k}^{-}\right) X_{i j}^{T}(t) P B K X_{k i}(t) \\
& \triangleq S_{21}(t)+S_{22}(t) .
\end{aligned}
$$

Note that

$$
\begin{aligned}
S_{21}(t)= & \frac{1}{N} \sum_{i=1}^{N} \sum_{j=1}^{N} \sum_{k=1}^{N}\left[l_{i k}^{+}\left(X_{i k}(t)+X_{k j}(t)\right)^{T} P B K X_{k i}(t)\right] \\
= & -\sum_{i=1}^{N} \sum_{k=1}^{N} l_{i k}^{+} X_{i k}^{T}(t) P B K X_{i k}(t) \\
& +\frac{1}{N} \sum_{i=1}^{N} \sum_{j=1}^{N} \sum_{k=1}^{N} l_{i k}^{+} X_{k j}^{T}(t) P B K X_{k i}(t) .
\end{aligned}
$$

Renaming the second sum in $S_{21}(t) k$ by $i$ and vice versa, we get

$$
S_{21}(t)=-\sum_{i=1}^{N} \sum_{k=1}^{N} l_{i k}^{+} X_{i k}^{T}(t) P B K X_{i k}(t)-S_{21}(t) .
$$


Thus,

$$
S_{21}(t)=-\frac{1}{2} \sum_{i=1}^{N} \sum_{j=1}^{N} l_{i j}^{+} X_{i j}^{T}(t) P B K X_{i j}(t)
$$

And, for the sum $S_{22}(t)$, we have

$$
\begin{aligned}
S_{22}(t)= & \frac{1}{N} \sum_{i=1}^{N} \sum_{j=1}^{N} \sum_{k=1}^{N} l_{i k}^{-} X_{i j}^{T}(t) P B K\left(X_{k j}(t)+X_{j i}(t)\right) \\
= & \frac{1}{N} \sum_{i=1}^{N} \sum_{j=1}^{N} \sum_{k=1}^{N} l_{i k}^{-} X_{i j}^{T}(t) P B K X_{k j}(t) \\
= & \frac{1}{N} \sum_{i=1}^{N} \sum_{j=1}^{N} l_{i i}^{-} X_{i j}^{T}(t) P B K X_{i j}(t) \\
& +\frac{1}{N} \sum_{i=1}^{N} \sum_{j=1}^{N} \sum_{k=1, k \neq i}^{N} l_{i k}^{-} X_{i j}^{T}(t) P B K X_{k j}(t) .
\end{aligned}
$$

Renaming the second sum in the above equation $i$ by $k$ and vice versa, we have

$$
\begin{aligned}
S_{22}(t) & =\frac{1}{N} \sum_{i=1}^{N} \sum_{j=1}^{N} l_{i i}^{-} X_{i j}^{T}(t) P B K X_{i j}(t) \\
& =\frac{1}{2 N} \sum_{i=1}^{N} \sum_{j=1}^{N}\left(l_{i i}^{-}+l_{j j}^{-}\right) X_{i j}^{T}(t) P B K X_{i j}(t) .
\end{aligned}
$$

Then $S_{2}(t)$ can be rewritten as

$$
S_{2}(t)=-\frac{1}{2} \sum_{i=1}^{N} \sum_{j=1}^{N}\left[l_{i j}^{+}-\frac{1}{N}\left(l_{i i}^{-}+l_{j j}^{-}\right)\right] X_{i j}^{T}(t) P B K X_{i j}(t) \text {. }
$$

Since $X_{i i}(t)=0$, then

$$
\begin{aligned}
S_{2}(t) & =-\frac{1}{2} \sum_{i=1}^{N} \sum_{j=1}^{N} \widetilde{l}_{i j} X_{i j}^{T}(t) P B B^{T} P X_{i j}(t) \\
& =-\sum_{i=1}^{N} \sum_{j=1}^{N} \widetilde{l}_{i j}\left[x_{i}^{T}(t) P B B^{T} P x_{i}(t)-x_{i}^{T}(t) P B B^{T} P x_{j}(t)\right] \\
& =\sum_{i=1}^{N} \sum_{j=1}^{N} \widetilde{l}_{i j} x_{i}^{T}(t) P B B^{T} P x_{j}(t) \\
& =X^{T}(t)\left[\widetilde{L} \otimes\left(P B B^{T} P\right)\right] X(t) .
\end{aligned}
$$

Substituting $S_{1}(t)$ and $S_{2}(t)$ into the derivative of $V(t)$ gives

$$
\begin{aligned}
\dot{V}(t) \leq & X^{T}(t)\left[\left(\Pi\left(\alpha I_{N}-c \widetilde{L}\right) \Pi\right) \otimes\left(P B B^{T} P\right)\right] X(t) \\
& -\beta X^{T}(t)\left(\Pi \otimes I_{n}\right) X(t) .
\end{aligned}
$$

Notice that $\widetilde{L}$ is a symmetric matrix; then there exists an orthogonal matrix $U \in R^{N \times N}$ such that $U \widetilde{L} U^{T}=\Lambda=$ $\operatorname{diag}\left(\lambda_{1}(\widetilde{L}), \lambda_{2}(\widetilde{L}), \ldots, \lambda_{N}(\widetilde{L})\right)$ with $\lambda_{1}(\widetilde{L})=0$. Therefore, the derivative of $V(t)$ satisfies

$$
\begin{aligned}
\dot{V}(t) \leq & Y^{T}(t)\left[\left(\alpha I_{N}-c \Lambda\right) \otimes\left(P B B^{T} P\right)\right] Y(t) \\
& -\beta X^{T}(t)\left(\Pi \otimes I_{n}\right) X(t) \\
= & \sum_{i=1}^{N}\left(\alpha-c \lambda_{i}(\widetilde{L})\right) y_{i}^{T}(t) P B B^{T} P y_{i}(t) \\
& -\beta X^{T}(t)\left(\Pi \otimes I_{n}\right) X(t) \\
\leq & \left(\alpha-c \lambda_{2}(\widetilde{L})\right) \sum_{i=1}^{N} y_{i}^{T}(t) P B B^{T} P y_{i}(t) \\
& -\beta X^{T}(t)\left(\Pi \otimes I_{n}\right) X(t) \\
\leq & -\beta \sum_{i=1}^{N}\left(x_{i}(t)-\bar{x}(t)\right)^{T}\left(x_{i}(t)-\bar{x}(t)\right),
\end{aligned}
$$

where $Y(t)=\left((U \Pi) \otimes I_{n}\right) X(t)=\left(y_{1}^{T}(t), \ldots, y_{N}^{T}(t)\right)^{T} \in$ $R^{n N}$ with $y_{1}(t)=\left(\xi_{1} \otimes I_{n}\right)\left(x_{i}(t)-\bar{x}(t)\right)=0, \bar{x}(t)=$ $(1 / N) \sum_{i=1}^{N} x_{i}(t)=\left(\xi^{T} \otimes I_{n}\right) X(t)$.

It is obvious that $\dot{V}(t) \leq 0$ and $\dot{V}(t)=0$ if and only if $x_{i}(t)=\bar{x}(t)$. By LaSalle's invariance principle, it follows that $x_{i}(t)-\bar{x}_{i}(t)$ exponentially converges to zero for all $i$ as time approaches infinity. Hence, the multiagent system (4) achieves global exponential consensus under the designed protocol. The proof is thus completed.

Remark 2. In terms of graph, the symmetrization operation in (12) amounts to replacing the edge directed from agent $i$ to agent $j$ by an undirected edge corresponding to the coupling coefficients and node strengths of agents $i$ and $j$. As a result, the consensus issue of a nonlinear multiagent system with a weighted directed communication graph $\mathscr{G}$ can be evaluated by the algebraic connectivity of a symmetric matrix associated with asymmetric Laplacian matrix $L$. From the above proof, the symmetrization operation in (12) can also deal with a time-varying Laplacian matrix.

Remark 3. Consider a special case of node balance; that is, $\sum_{i=1}^{N} l_{i j}=0$. Then we have $\widetilde{L}=L^{+}$and the following result.

Corollary 4. Suppose that (H1) and (H2) hold. If

$$
c \lambda_{2}\left(L^{+}\right) \geq \alpha
$$

then multiagent system (1) with node balance achieves global exponential consensus under the designed controller (2) and $K=B^{T} P$.

It is noted that $\lambda_{2}\left(L^{+}\right)>0$ under (H1). Then the consensus of system (1) can be guaranteed by a sufficiently large coupling strength $c$. 


\section{An Example}

In this section, a multiagent system consisting of five identical agents will be constructed to demonstrate the efficiency of the result proposed in the previous section. The agent dynamics can be described by

$$
f\left(x_{i}\right)=A x_{i}+g\left(x_{i}\right)
$$

where $x_{i}=\left(x_{i 1}, x_{i 2}, x_{i 3}, x_{i 4}\right)^{T}, g\left(x_{i}\right)=$ $\left(0,0,0,-(1 / 3) \sin \left(x_{i 3}\right)\right)^{T}$, and

$$
A=\left(\begin{array}{cccc}
0 & 1 & 0 & 0 \\
-48.6 & -1.25 & 48.6 & 0 \\
0 & 0 & 0 & 10 \\
1.95 & 0 & -1.95 & 0
\end{array}\right)
$$

The input matrix is defined as $B=(0,20,0,0)^{T}$ and Laplacian matrix is chosen as

$$
L=\left(\begin{array}{ccccc}
2 & -2 & 0 & 0 & 0 \\
0 & 2 & -2 & 0 & 0 \\
0 & 0 & 4 & -4 & 0 \\
0 & 0 & 0 & 2 & -2 \\
0 & 0 & 0 & 0 & 0
\end{array}\right)
$$

By calculation, we have $\lambda_{2}(\widetilde{L})=0.1728$. Also notice that

$$
\begin{aligned}
& \left(g\left(x_{i}\right)-g\left(x_{j}\right)\right)^{T} P X_{i j}(t) \\
& \quad \leq\left\|\left(g\left(x_{i}\right)-g\left(x_{j}\right)\right)^{T} P Q^{-1 / 2}\right\| \cdot\left\|X_{i j}(t) Q^{1 / 2}\right\| \\
& \quad \leq \gamma\left\|X_{i j}^{T} P Q^{-1 / 2}\right\| \cdot\left\|X_{i j}(t) Q^{1 / 2}\right\| \\
& \quad \leq \frac{1}{2} X_{i j}^{T}(t)\left(\gamma^{2} P Q^{-1} P+Q\right) X_{i j}(t),
\end{aligned}
$$

where $\gamma=1 / 3$ and $Q \in R^{n \times n}$ is an arbitrary positive definite matrix to be determined. Substituting (35) into (H2) gives the following linear matrix inequality:

$$
A^{T} P+P A-2 \alpha P B B^{T} P+\gamma^{2} P Q^{-1} P+Q+2 \beta I_{n} \leq 0 .
$$

Solving (36) gives

$$
P=\left(\begin{array}{cccc}
18.2759 & 0.3099 & -10.8809 & 28.4167 \\
0.3099 & 0.0228 & -0.1837 & 0.4840 \\
-10.8809 & -0.1837 & 8.4982 & -14.1034 \\
28.4167 & 0.4840 & -14.1034 & 75.9731
\end{array}\right)
$$

and $\alpha=0.57$ by setting $\beta=0.01$ and $Q=I_{4}$. Then, according to Theorem 1, the global consensus is achieved if $c>3.3$. The state responses are depicted in Figure 1 with $c=3.5$.

\section{Conclusions}

This paper has investigated the global consensus problem of multiagent systems with asymmetrically coupled nonidentical agents. By employing a Lyapunov function, a criterion of global exponential consensus has been derived under the
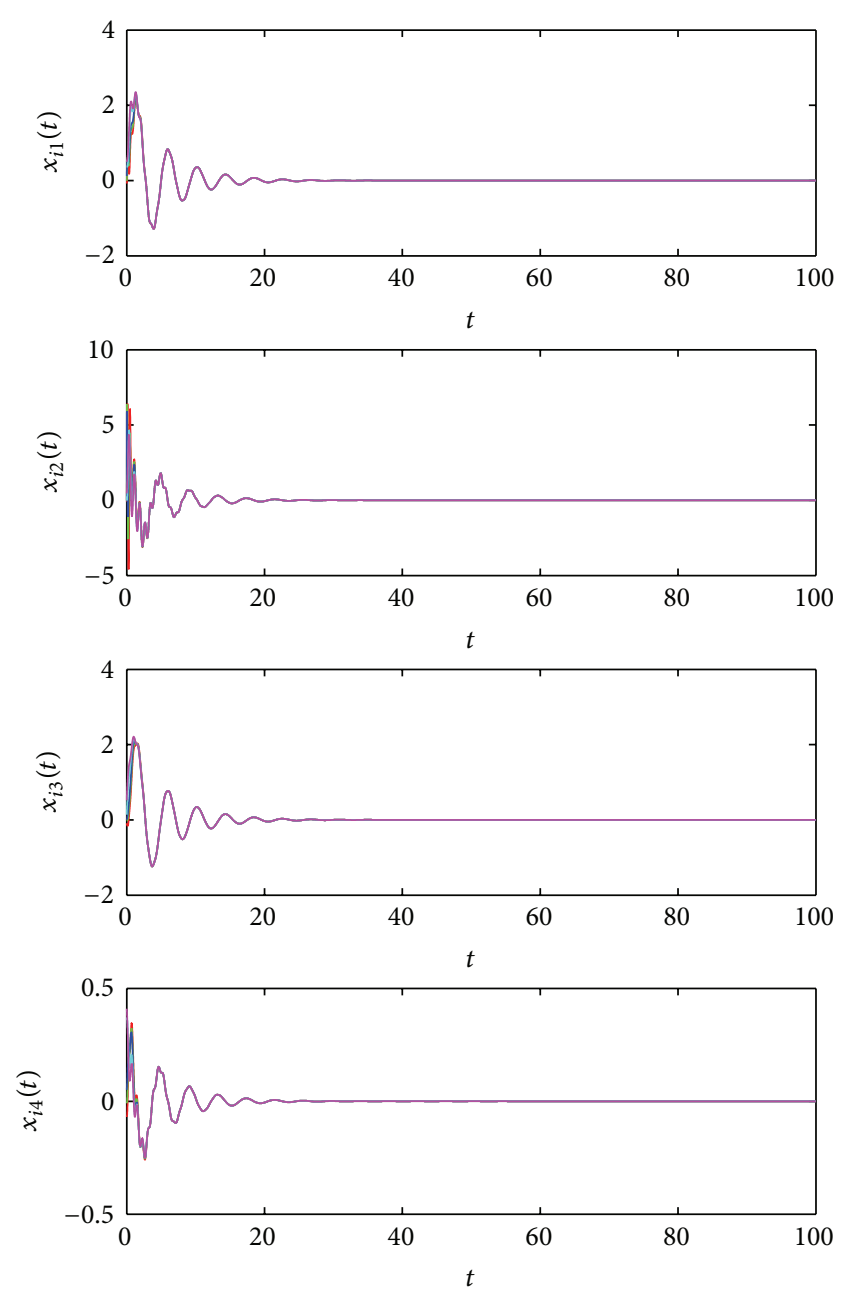

FIGURE 1: The state responses of the considered multiagent system.

designed consensus protocol. The presented framework is quite simple and powerful, without assuming the symmetry or irreducibility of the Laplacian matrix, just evaluating the algebraic connectivity of a symmetric matrix derived by the Laplacian matrix. A numerical simulation is given to show the effectiveness of the analytical result.

\section{Conflict of Interests}

The authors declare that there is no conflict of interests regarding the publication of this paper.

\section{Acknowledgments}

This work is supported by the National Natural Science Foundations of China nos. 61104119, 61004106, and 61340015, the Science and Technology Innovation Talents Project of Henan University under Grant 13HASTIT044, the Young Core Instructor Foundation from Department of Education of Henan Province under Grant 2011GGJS-054, and the Fundamental Research Funds for the Central Universities nos. YWF-13-T-RSC-023 and YWF-13-A02-17. 


\section{References}

[1] S. Kar and J. M. F. Moura, "Sensor networks with random links: topology design for distributed consensus," IEEE Transactions on Signal Processing, vol. 56, no. 7, pp. 3315-3326, 2008.

[2] Y. Zhuang, Z. Wang, H. Yu, W. Wang, and S. Lauria, "A robust extended $H_{\infty}$ filtering approach to multi-robot cooperative localization in dynamic indoor environments," Control Engineering Practice, vol. 21, no. 7, pp. 953-961, 2013.

[3] T. Vicsek, A. Czirók, E. Ben-Jacob, I. Cohen, and O. Shochet, "Novel type of phase transition in a system of self-deriven particles," Physical Review Letters, vol. 75, pp. 1226-1229, 1995.

[4] A. Jadbabaie, J. Lin, and A. S. Morse, "Coordination of groups of mobile autonomous agents using nearest neighbor rules," IEEE Transactions on Automatic Control, vol. 48, no. 6, pp. 988-1001, 2003.

[5] R. Olfati-Saber and R. M. Murray, "Consensus problems in networks of agents with switching topology and time-delays," IEEE Transactions on Automatic Control, vol. 49, no. 9, pp. 15201533, 2004.

[6] L. Moreau, "Stability of multiagent systems with time-dependent communication links," IEEE Transactions on Automatic Control, vol. 50, no. 2, pp. 169-182, 2005.

[7] W. Ren and R. W. Beard, "Consensus seeking in multiagent systems under dynamically changing interaction topologies," IEEE Transactions on Automatic Control, vol. 50, no. 5, pp. 655661, 2005.

[8] M. Cao, A. S. Morse, and B. D. O. Anderson, "Reaching a consensus in a dynamically changing environment: a graphical approach," SIAM Journal on Control and Optimization, vol. 47, no. 2, pp. 575-600, 2008.

[9] Y. Hong, L. Gao, D. Cheng, and J. Hu, "Lyapunov-based approach to multiagent systems with switching jointly connected interconnection," IEEE Transactions on Automatic Control, vol. 52, no. 5, pp. 943-948, 2007.

[10] P. Lin and Y. Jia, "Further results on decentralised coordination in networks of agents with second-order dynamics," IET Control Theory and Applications, vol. 3, no. 7, pp. 957-970, 2009.

[11] G. Xie and L. Wang, "Consensus control for a class of networks of dynamic agents," International Journal of Robust and Nonlinear Control, vol. 17, no. 10-11, pp. 941-959, 2007.

[12] W. Ren, K. L. Moore, and Y. Chen, "High-order and model reference consensus algorithms in cooperative control of multivehicle systems," Journal of Dynamic Systems, Measurement and Control, Transactions of the ASME, vol. 129, no. 5, pp. 678-688, 2007.

[13] Z. Ji, Z. Wang, H. Lin, and Z. Wang, "Interconnection topologies for multi-agent coordination under leader-follower framework," Automatica, vol. 45, no. 12, pp. 2857-2863, 2009.

[14] W. Yu, G. Chen, W. Ren, J. Kurths, and W. X. Zheng, "Distributed higher order consensus protocols in multiagent dynamical systems," IEEE Transactions on Circuits and Systems I: Regular Papers, vol. 58, no. 8, pp. 1924-1932, 2011.

[15] Z. Wang, Y. Wang, and Y. Liu, "Global synchronization for discrete-time stochastic complex networks with randomly occurred nonlinearities and mixed time delays," IEEE Transactions on Neural Networks, vol. 21, no. 1, pp. 11-25, 2010.

[16] W. Xiong, D. W. C. Ho, and Z. Wang, "Consensus analysis of multiagent networks via aggregated and pinning approaches," IEEE Transactions on Neural Networks, vol. 22, no. 8, pp. 12311240, 2011.
[17] Z. Wang, D. Ding, H. Dong, and H. Shu, " $H_{\infty}$ consensus control for multi-agent systems with missing measurements: the finitehorizon case," Systems \& Control Letters, vol. 62, pp. 827-836, 2013.

[18] Q. Wang, H. Gao, F. Alsaadi, and T. Hayat, "An overview of consensus problems in constrained multi-agent coordination," Systems Science and Control Engineering, vol. 2, no. 1, pp. 275284, 2014.

[19] M. Arcak, "Passivity as a design tool for group coordination," IEEE Transactions on Automatic Control, vol. 52, no. 8, pp. 13801390, 2007.

[20] L. I. N. Zhiyun, B. Francis, and M. Maggiore, "State agreement for continuous-time coupled nonlinear systems," SIAM Journal on Control and Optimization, vol. 46, no. 1, pp. 288-307, 2007.

[21] J. Cortés, "Distributed algorithms for reaching consensus on general functions," Automatica, vol. 44, no. 3, pp. 726-737, 2008.

[22] Z. Qu, J. Wang, and R. A. Hull, "Cooperative control of dynamical systems with application to autonomous vehicles," IEEE Transactions on Automatic Control, vol. 53, no. 4, pp. 894911, 2008.

[23] W. Yu, G. Chen, M. Cao, and J. Kurths, "Second-Order consensus for multiagent systems with directed topologies and nonlinear dynamics," IEEE Transactions on Systems, Man, and Cybernetics B: Cybernetics, vol. 40, no. 3, pp. 881-891, 2010.

[24] G. Wen, Z. Duan, Z. Li, and G. Chen, "Consensus and its 12-gain performance of multi-agent systems with intermittent information transmissions," International Journal of Control, vol. 85, no. 4, pp. 384-396, 2012.

[25] L. Wang and Q. -G. Wang, "Average contraction and synchronization of complex switched networks," Journal of Physics A, vol. 45, pp. 205-210, 2012.

[26] W. Yu, G. Chen, and M. Cao, "Consensus in directed networks of agents with nonlinear dynamics," IEEE Transactions on Automatic Control, vol. 56, no. 6, pp. 1436-1441, 2011.

[27] Z. Li, Z. Duan, G. Chen, and L. Huang, "Consensus of multiagent systems and synchronization of complex networks: a unified viewpoint," IEEE Transactions on Circuits and Systems, vol. 57, pp. 213-224, 2010.

[28] J. H. Seo, H. Shim, and J. Back, "Consensus of high-order linear systems using dynamic output feedback compensator: low gain approach," Automatica, vol. 45, no. 11, pp. 2659-2664, 2009.

[29] C. W. Wu and L. O. Chua, "Synchronization in an array of linearly coupled dynamical systems," IEEE Transactions on Circuits and Systems I: Fundamental Theory and Applications, vol. 42, no. 8, pp. 430-447, 1995.

[30] V. N. Belykh, I. V. Belykh, and M. Hasler, "Connection graph stability method for synchronized coupled chaotic systems," Physica D: Nonlinear Phenomena, vol. 195, no. 1-2, pp. 159-187, 2004. 


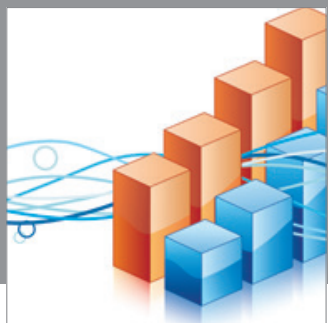

Advances in

Operations Research

mansans

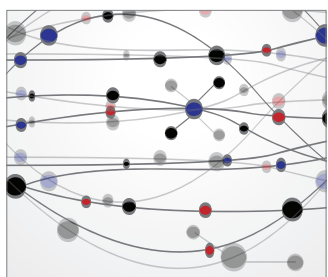

The Scientific World Journal
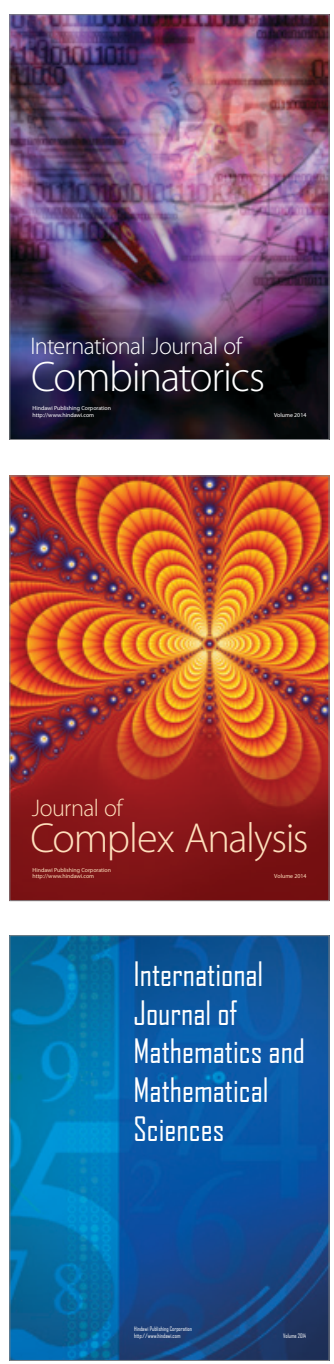
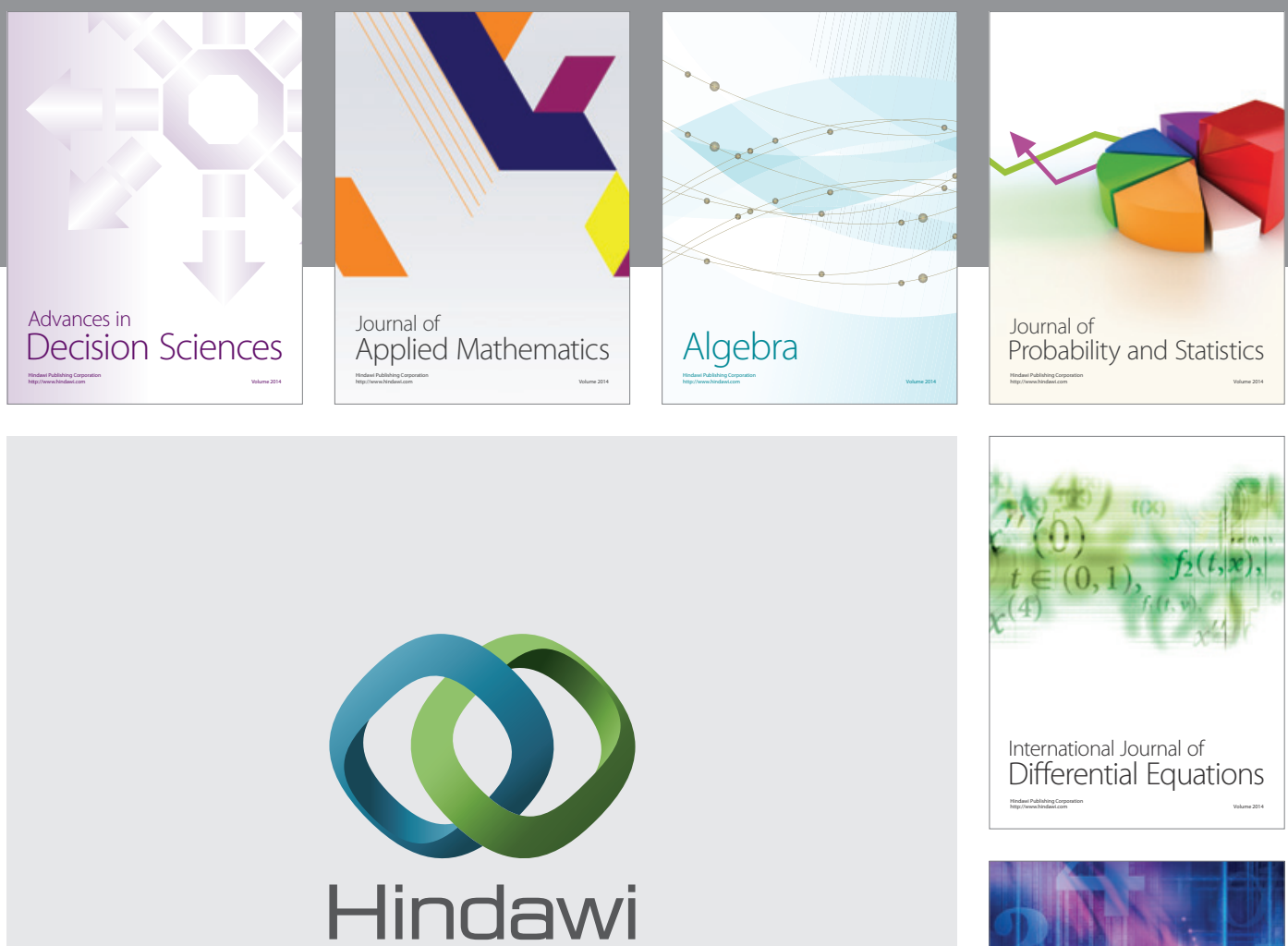

Submit your manuscripts at http://www.hindawi.com
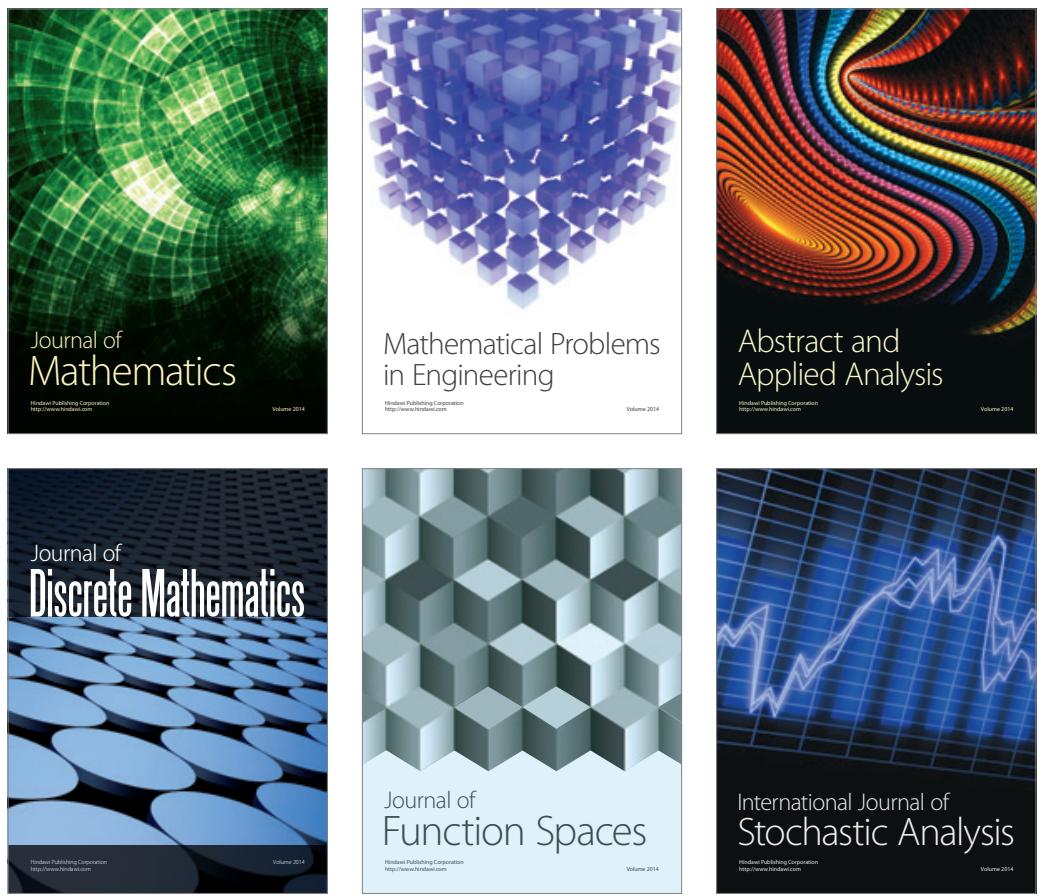

Journal of

Function Spaces

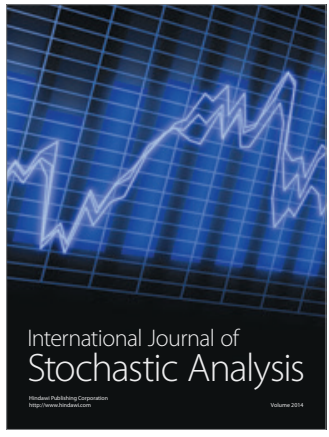

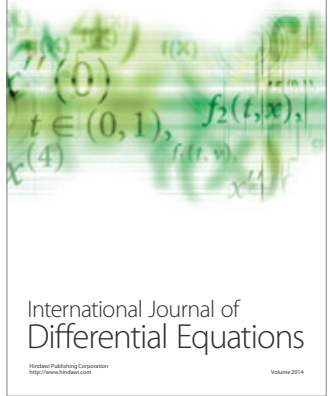
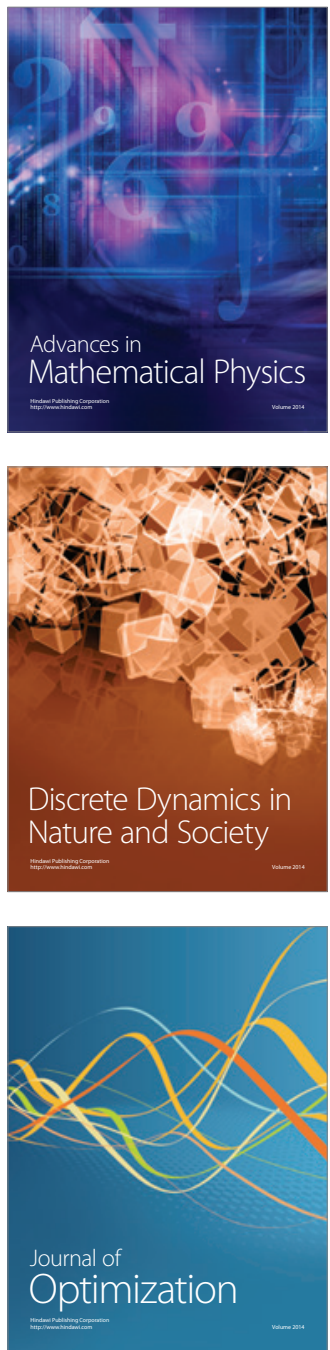